\title{
O DESEMPENHO EM TECNOLOGIAS DIGITAIS PARA APRENDIZAGEM: UM ESTUDO COM UNIVERSITÁRIOS
}

\author{
THE PERFORMANCE IN DIGITAL TECHNOLOGIES FOR LEARNING: \\ A STUDY WITH UNIVERSITY
}

\section{EL RENDIMIENTO EN TECNOLOGÍAS DIGITALES PARA EL APRENDIZAJE: UN ESTUDIO CON UNIVERSITARIOS}

\author{
Wagner Roberto Dias Nascimento ${ }^{1}$; Ana Cristina Salviato-Silva², \\ Betânia Alves Veiga Dell' Agli
}

\section{RESUMO}

O objetivo deste artigo é analisar a percepção que os universitários têm sobre a utilização de recursos de tecnologia digital de informação e comunicação (TDIC) para sua aprendizagem. Participaram do estudo 308 alunos de um curso presencial em uma universidade pública brasileira. Foi utilizado um questionário online como instrumento de coleta, em que seus dados foram analisados com uma abordagem quantitativa. Identificou-se que a tecnologia digital de informação e comunicação foi percebida como grande apoiadora nos estudos, porém havia alta heterogeneidade de sua apropriação nos estudos por calouros ou veteranos e mais da metade indicava que raramente ou nunca recebiam incentivo dos professores para a utilizarem. A apropriação desse recurso pode ampliar o desempenho acadêmico e reduzir a evasão escolar.

PALAVRAS-CHAVE: Educação Superior; Tecnologia Educacional; Evasão Escolar.

\section{ABSTRACT}

The purpose of this article is to analyze the perception that university students have about the use of digital information and communication technology (TDIC) resources for their learning. A total of 308 students attending a face-to-face course at a Brazilian public university participated in the study. An online questionnaire was used as a collection tool, in which the data were analyzed with a quantitative approach. It was identified that digital information and communication technology was perceived as a great support in the studies, but there was a high heterogeneity of their appropriation in the studies by freshmen or veterans, and more than half indicated that they rarely or never received an incentive from the teachers to use it. The appropriation of this resource can increase academic performance and reduce school dropout.

\footnotetext{
${ }^{1}$ Mestre em educação, ambiente e sociedade - Centro Universitário das Faculdades Associadas de Ensino (UNIFAE) - São João da Boa Vista, SP - Brasil. Analista de Sistemas, Especialista em Gestão Estratégica de Tecnologia - Centro Universitário das Faculdades Associadas de Ensino (UNIFAE) - São João da Boa Vista, SP Brasil. E-mail: wagnernnascimento@gmail.com

2 Doutora e Mestre em Linguística e Língua Portuguesa - Universidade Estadual Paulista Júlio de Mesquita Filho (UNESP) - São Paulo, SP - Brasil. Docente - Centro Universitário das Faculdades Associadas de Ensino (UNIFAE) São João da Boa Vista, SP - Brasil. E-mail: anasalviato@uol.com.br

${ }^{3}$ Doutora em Educação - Universidade Estadual de Campinas (UNICAMP) - Campinas, SP - Brasil

Docente - Centro Universitário das Faculdades Associadas de Ensino (UNIFAE) - São João da Boa Vista, SP Brasil. E-mail: betania@fae.br
}

Submetido em: 24/01/2018 - Aceito em: 27/08/2018

(C) ETD-Educação Temática Digital Campinas, SP $\quad$ v.21 n.1 $\quad$ p.182-201 jan./mar. 2019 
KEYWORDS: Education, Higher; Educational Technology; Student Dropouts.

\section{RESUMEN}

El objetivo de este artículo es analizar la percepción que tienen los estudiantes universitarios sobre el uso de los recursos de la tecnología de información y comunicación digital (TDIC) para su aprendizaje. Un total de 308 estudiantes que asistieron a un curso presencial en una universidad pública brasileña participaron en el estudio. Se utilizó un cuestionario en línea como una herramienta de recopilación, en la que los datos se analizaron con un enfoque cuantitativo. Se identificó que la tecnología de información y comunicación digital se percibía como un gran apoyo en los estudios, pero había una gran heterogeneidad de su apropiación en los estudios por estudiantes de primer año o veteranos, y más de la mitad indicaba que rara vez o nunca recibían un incentivo de parte de los maestros para usar eso. La apropiación de este recurso puede aumentar el rendimiento académico y reducir el abandono escolar.

PALAVRAS-CLAVE: Educación universitaria; Tecnología Educativa; Evasión Escolar

\section{INTRODUÇÃO}

No final do século XIX, a pedagogia do método intuitivo ou lições de coisas foi introduzida no Brasil. A principal catalisadora para essa mudança foi a Revolução Industrial, pois trouxe novas exigências sociais para escola e, ao mesmo tempo, permitiu a produção em série de novos materiais didáticos, tais como mapas, diagramas, objetos de madeira, louças e vidros. Grande parte desse modelo permanece até hoje (SAVIANI, 2008).

Atualmente, os recursos das tecnologias digitais de informação e comunicação (TDIC) estão inseridos no cotidiano de nossa sociedade. As tradicionais fontes de consulta em mídia impressa, tais como livros, enciclopédias e jornais passaram a disputar espaço com sítios na internet, enciclopédias eletrônicas e ferramentas de busca tais como o Google ou Yahoo. $\mathrm{Na}$ cibercultura, a aprendizagem ao longo da vida dissolve a divisão entre período de aprender e de trabalhar, promove uma inteligência coletiva e "amplifica uma profunda relação com o saber" (LEVY, 1999, p. 172).

Muitos dos jovens que atualmente estão iniciando seus estudos em nível superior nasceram após o surgimento da internet comercial e, desde muito cedo, foram expostos aos seus recursos. Nos chamados "nativos-digitais", há expectativa que possuam aptidão natural para sua utilização (PRENSKY, 2001; PALFREY; GASSER, 2008; JONES et al., 2010). Segundo dados do Censo da Educação Superior Brasileira, no ano de 2013, havia 2.391 instituições e 2.742.950 de ingressantes no ensino superior (INEP, 2015). A cada semestre, há mais universitários nativos-digitais.

A ampliação das capacidades cognitivas atribuídas no início do século XX para a escrita, atualmente pode ser aplicada aos recursos tecnológicos. Naquela época considerava-se,

(C) ETD-Educação Temática Digital Campinas, SP $\quad$ v.21 n.1 $\quad$ p.182-201 jan./mar. 2019 
o domínio desse sistema complexo de signos fornece novo instrumento de pensamento (na medida em que aumenta a capacidade de memória, registro de informações etc.). Propicia diferentes formas de organizar a ação e permite um outro tipo de acesso ao patrimônio da cultura humana (REGO, 2010, p.24).

Por outro lado, estudos sobre a internet indicam que jovens universitários são mais ativos em atividades de lazer e comunicação, enquanto os alunos mais velhos são mais focados em atividades relacionadas com fins acadêmicos (GROS; GARCIA; ESCOFET, 2012). A gestão deste cenário pode beneficiar-se dos conceitos da educomunicação, que estudam e propõem maneiras pelos quais os ecossistemas educativos podem apropriar-se dos recursos de comunicação existentes a fim de atingir seus objetivos (SOARES, 2002).

Partindo da premissa que os jovens brasileiros utilizam tecnologias digitais de informação e comunicação para os estudos (CAVASSANI, 2016), a problematização conduz até a questão: a apropriação desses recursos é uniforme? O objetivo deste artigo é analisar a percepção que os universitários têm acerca da utilização de recursos de tecnologia digital de informação e comunicação para sua aprendizagem.

\section{MÉTODO}

Trata-se de um estudo de campo, não experimental, transversal, descritivo e de abordagem quantitativa (SAMPIERI; COLLADO; LUCIO, 2013). Para alcançar o objetivo proposto, foram definidos cinco objetivos específicos, que estão associados às hipóteses $(H)$, respectivamente.

Foram submetidas a testes estatísticos as hipóteses a seguir: $(\mathrm{H} 1)$ a maioria dos estudantes tem percepção de que o uso de recursos computacionais contribui positivamente em seu processo de aprendizagem; $(\mathrm{H} 2)$ há diferenças de desempenho relacionadas à idade, ao sexo, à experiência universitária, ao turno (noturno ou integral) ou cursos na área de Tl; (H3) a insegurança ou desconforto na utilização de computadores para estudar diminui à medida que aumenta o número de semestres cursados; $(\mathrm{H} 4)$ o desempenho dos estudantes no uso de TDIC concentra-se em ferramentas básicas e comunicação; (H5) os estudantes recebem incentivo dos professores para utilizar o computador para estudar e encontram condições de infraestrutura favoráveis. 


\subsection{Participantes}

Foram convidados a participar do presente estudo discentes de um curso presencial de bacharelado interdisciplinar em ciência e tecnologia em uma universidade pública brasileira. A instituição oferece biblioteca (com notebooks) e laboratórios de informática providos de computadores do tipo desktop, com monitores de 19 polegadas, teclado e mouse. Também há redes sem fio nas salas de aula e espaços disponíveis aos alunos, de modo que o estudante possa utilizar equipamentos institucionais ou próprios para acesso à internet.

Foi adotado como critério de inclusão estarem presentes no dia previsto para coleta dos dados, ou seja, o instrumento não foi disponibilizado para mesma turma em nova oportunidade. $\mathrm{O}$ critério de exclusão corresponde aos discentes com idade menor que dezoito anos.

O tamanho da amostra foi calculado a partir da população de 900 estudantes, margem de erro de $5 \%$ e nível de confiança de $95 \%$, o que resultou no tamanho da amostra de 269 participantes (COCHRAN, 2007). Trezentos e onze discentes aceitaram participar e responderam completamente a pesquisa; entre eles, três mulheres com idade 17 anos que atenderam ao critério de exclusão e suas respostas foram dispensadas, de maneira que os resultados apoiam-se em 308 participantes.

\subsection{Instrumentos para coleta de dados}

Para atender aos objetivos da pesquisa, foi utilizado como instrumento um questionário autoaplicado, em formato eletrônico, acessível por meio de um link de internet.

A primeira parte do instrumento consiste na apresentação da pesquisa e o Termo de Consentimento Livre e Esclarecido. A segunda parte, contêm questões de caracterização do participante que mantém seu anonimato e a terceira e última, disponibiliza 57 questões fechadas, correspondentes à Escala de Desempenho em Tecnologias (EDTEC), um instrumento construído e validado no Brasil para avaliar os estudantes que utilizam tecnologias de informação e comunicação em suas estratégias educacionais para o aprendizado (JOLY; MARTINS, 2006).

Este instrumento foi construído a partir das competências preconizadas pelo International Society for Technology in Education (ISTE, 1998) e suas questões são agrupadas nos fatores "ferramentas básicas e de comunicação", "conceitos e ferramentas de produtividade" e "ferramentas de solução de problemas".

(C) ETD- Educação Temática Digital Campinas, SP $\quad$ v.21 n.1 $\quad$ p.182-201 jan./mar. 2019 


\subsection{Procedimentos para coleta de dados}

A presente pesquisa foi autorizada pela Direção do Campus e aprovada pelo Comitê de Ética em Pesquisa com o parecer número 1.816.244. Na sequência, identificaram-se os docentes responsáveis por disciplinas nos laboratórios de informática, que foram contatados e autorizaram um período em torno de quinze minutos, previamente agendado para aplicação do instrumento.

O questionário foi aplicado no início do semestre letivo, pois havia a exigência que fosse distante do período de provas. Conforme combinado, o pesquisador compareceu no início das aulas de catorze turmas, dos períodos noturno e integral, apresentou a pesquisa e forneceu o link para acesso ao instrumento.

Não houve contato adicional com o pesquisador durante o preenchimento. O tempo médio para respondê-lo foi de catorze minutos e após a coleta, o link foi desativado para impossibilitar novos acessos.

\subsection{Análise dos dados}

Os dados armazenados no site foram exportados para uma planilha eletrônica em formato Microsoft Excel e examinados em conjunto com a ferramenta estatística ActionStat (EQUIPE ESTATCAMP, 2014).

Esse procedimento permitiu que os dados cadastrais fossem visualizados e a Escala de Desempenho em Tecnologias para fins educacionais (EDTEC) fosse calculada para cada voluntário. Para as respostas "nunca", às vezes", "muitas vezes" e "sempre" foram atribuídos os valores 1, 2, 3 e 4, respectivamente. Os resultados foram padronizados em percentuais entre o máximo possível e o valor efetivamente encontrado, para que os fatores pudessem ser comparados entre si.

(C) ETD- Educação Temática Digital Campinas, SP $\quad$ v.21 n.1 $\quad$ p.182-201 jan./mar. 2019 


\section{RESULTADOS E DISCUSSÃO}

Ao se examinar os dados sobre turno e sexo, identificou-se uma distribuição uniforme, apresentada na tabela 1 :

Tabela 1. Distribuição por sexo entre os turnos

\begin{tabular}{lccc}
\hline & Integral & Noturno & Total \\
\hline Feminino & $23 \%$ & $21 \%$ & $44 \%$ \\
Masculino & $29 \%$ & $27 \%$ & $56 \%$ \\
\hline Total & $52 \%$ & $48 \%$ & $100 \%$ \\
\hline
\end{tabular}

Fonte: dados da pesquisa.

O curso de bacharelado interdisciplinar em ciência e tecnologia possui seis semestres e é o ciclo básico para ingresso nas engenharias química, de minas e ambiental. Tradicionalmente, espera-se que cursos na área de exatas possuam baixa concentração de mulheres em relação aos homens. Isso foi apurado pelo método Kruskal-Wallis rank sum test, definido como um teste não paramétrico que permite identificar se há diferença estatisticamente relevante em pelo menos uma das populações observadas. O resultado aponta P-valor de 0,8816, superior à margem de erro de 5\%, de maneira que essa hipótese não encontrou suporte estatístico nos dados coletados.

A exploração da média etária da amostra apontou que está posicionada em vinte anos, e o 3ㅇquartil dos turnos integral e noturno encontra-se respectivamente em 21 e 22,75 anos. Nesse caso, há evidência estatística para a hipótese de diferença etária entre os turnos, conforme apurado pelo método Kruskal-Wallis rank sum test, com P-valor de 0,0001, a idade dos alunos turno noturno é maior.

As especificidades próprias nos modos de se relacionar com e por meio da tecnologia introduziram na cultura contemporânea características originadas do ciberespaço, interferindo na linguagem, aprendizagem e na formação de professores (CONTE; MARTINI, 2015).

A internet favoreceu o surgimento de novas opções de trabalho, estudo e lazer. 0 tempo para cada ciclo de mudanças tem se tornado cada vez mais curto, de modo que a aprendizagem ao longo da vida é uma necessidade e a globalização requer o desenvolvimento da cidadania global, uma vez que mesmo países independentes compartilham dos mesmos 
desafios climáticos, epidemiológicos ou econômicos (UNESCO, 2016).

Entretanto, os processos educacionais apropriam-se das novas mídias de comunicação em velocidade muito pequena, dificultando inovações e integração de dispositivos técnicos (BÉVORT; BELLONI, 2009). Houve ampliação dos horizontes do acesso à informação, "todavia, o que se constata é a predominância da difusão de dados e informações e não de conhecimentos" (GADOTTI, 2000, p. 7).

A EDTEC pode ser utilizada para observar o desempenho na utilização da tecnologia para fins educacionais, enquanto que seus fatores nos dão pistas sobre como está o desenvolvimento particular nas áreas básicas ou comunicação, produtividade e solução de problemas. Realizado seu cálculo, o valor apurado foi submetido a testes de normalidade pelos métodos Anderson - Darling $(P$-valor $=0,5614)$, Kolmogorov - Smirnov (P-valor $=$ 0,6433), Shapiro - Wilk (P-valor $=0,2513$ ) e Ryan - Joiner ( $P$-valor $=0,4659)$. Os valores encontrados confirmam a hipótese de que os dados são modelados por uma distribuição normal e, portanto é possível a utilização dos métodos de Analysis of Variance (ANOVA) para "avaliar se diferenças observadas entre as médias das amostras são estatisticamente significantes" (LAPPONI, 2005, p.380).

Com o propósito de avaliar a $\mathrm{H} 1$, as respostas para a afirmação "aprendo mais rápido quando uso computador" revelam que apenas $3 \%$ entendem que a utilização de computadores nunca auxilia a aprendizagem, enquanto que $27 \%$ relatam "sempre", $40 \%$, "muitas vezes" e $30 \%$, "às vezes".

Há forte percepção que as tecnologias de informação e comunicação são facilitadoras do processo de aprendizagem. Os universitários pesquisados estão em uma faixa etária em que essa crença predomina e encaram a tecnologia como parte de suas vidas profissional e social (ROBERTO; FIDALGO; BUCKINGHAM, 2015).

No processo de ensino e de aprendizagem, a tecnologia deve ser tratada como mais uma ferramenta disponível. A percepção de $70 \%$ dos jovens de que ela auxilia "às vezes" ou "muitas vezes" sugere que deve existir alguma gestão desse processo comunicacional. Nesse sentido, a educomunicação favorece a emergência de zonas de desenvolvimento proximal, por meio de convívio entre pares, interação com o mais experiente e o suporte tecnológico (ARRUDA et al., 2012).

O interesse na utilização de computadores para os estudos está associado à expectativa de que essa ferramenta aumentará o desempenho nas atividades relacionadas à aprendizagem (MARCELA; DEL, 2015), conjuntamente às características de sexo, de idade, de

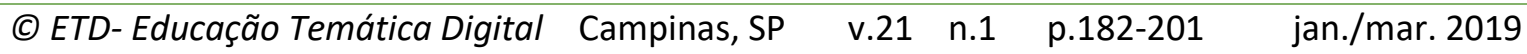


experiência, influência social e expectativa de esforço, essa última relacionada ao desempenho operacional na tecnologia empregada (VENKATESH et al., 2003).

Por fim, a hipótese (H1) foi confirmada, ou seja, 97\% dos estudantes relatam que estudar utilizando o computador contribui positivamente para seu processo de aprendizagem, ainda que em graus diversos.

Com o propósito de avaliar a hipótese $(\mathrm{H} 2)$, ao se examinar a EDTEC em relação à idade, não foi encontrada evidência estatística para a hipótese de que há variação do valor EDTEC com relação a ela, uma vez que tabela ANOVA efeito fixo apresenta P-valor 0,3879, superior à margem de erro 0,05. Portanto, essa hipótese foi rejeitada.

No enfoque relacionado ao sexo, a análise descritiva do desempenho em tecnologia de informação para fins educacionais apontou que há pouquíssima diferença entre os homens e mulheres, conforme figura 1 :

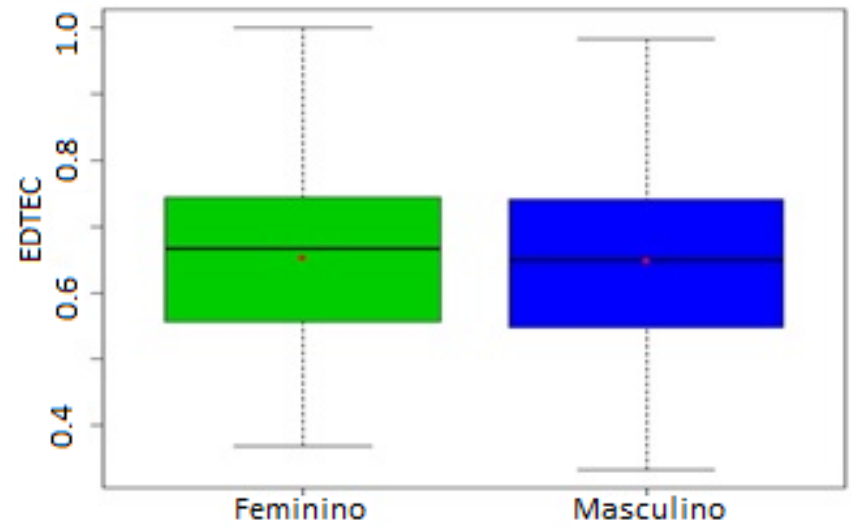

Figura 1 - Desempenho por sexo Fonte: dados da pesquisa.

Nota-se que a amplitude no desempenho é muito alta, ambos com valores mínimos abaixo de $37 \%$. O intervalo entre o primeiro e terceiro quartil encontra-se entre $54 \%$ e $74 \%$, o que aponta que existe grande heterogeneidade entre os participantes, com alunos que utilizam muito mais os recursos computacionais para fins de aprendizagem do que outros colegas de estudos.

Entretanto, homens e mulheres têm resultados similares inclusive nas suas diferenças de desempenho, de modo que a hipótese de haver diferenças relacionadas ao sexo foi rejeitada, com P-valor 0,866.

(C) ETD- Educação Temática Digital Campinas, SP $\quad$ v.21 n.1 $\quad$ p.182-201 jan./mar. 2019 
Para se investigar se o padrão de desempenho seria diferente após a experiência universitária, aproveitou-se de que o período da coleta aconteceu na primeira semana de aulas do semestre, desse modo os resultados entre os calouros e veteranos foi comparado.

Há 112 calouros na amostra e os veteranos estão distribuídos entre dois e dez semestres. Importante destacar que por se tratar de um estudo transversal, não foi feito o acompanhamento do mesmo indivíduo ao longo dos anos. Os resultados encontrados no cenário atual estão apresentados na figura 2:

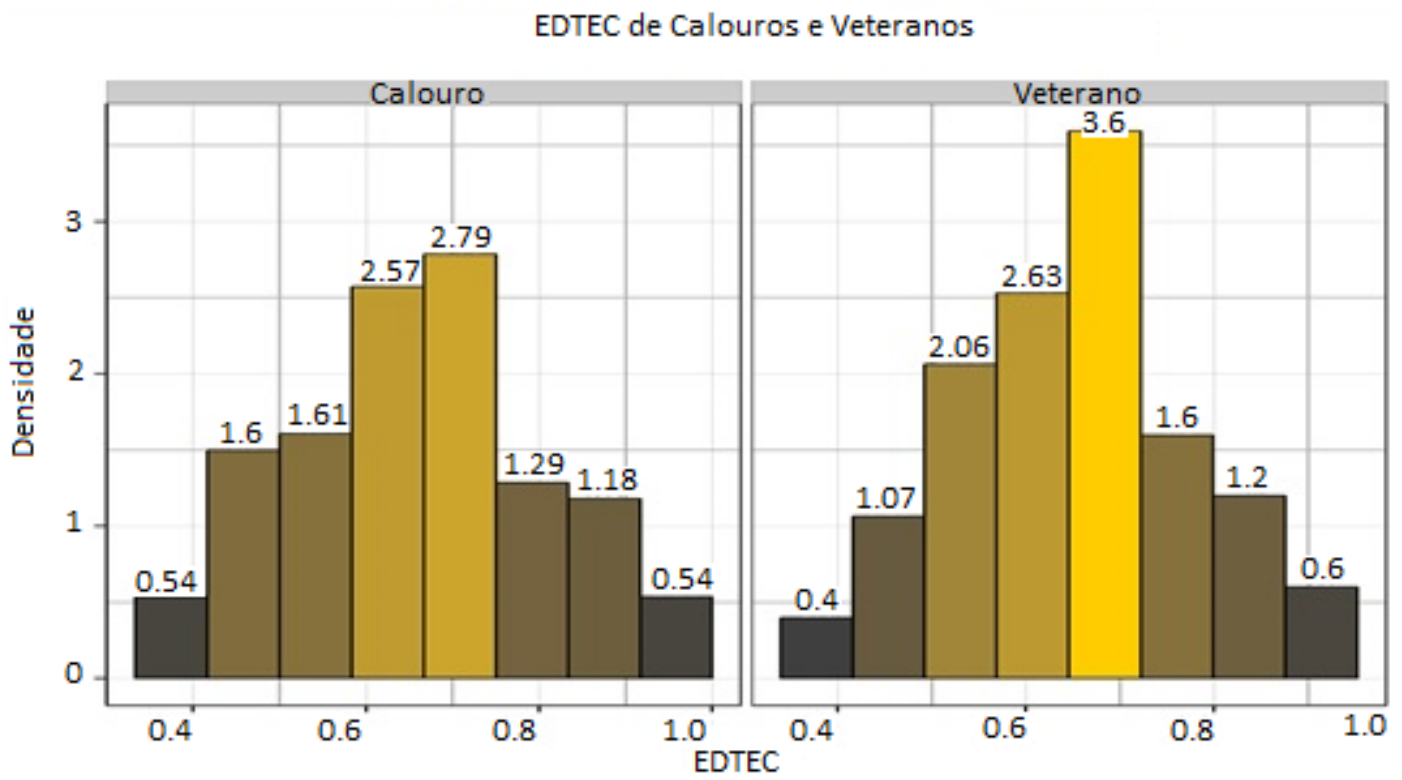

Figura 2 - Desempenhos de calouros e veteranos Fonte: dados da pesquisa.

Nota-se pela distribuição de densidades que a proporção de discentes com EDTEC acima de $74 \%$ (3 quartil) manteve-se praticamente estável entre calouros e veteranos. Observa-se, também, nos veteranos, uma maior concentração no centro da distribuição e a manutenção de indivíduos nas extremidades com pior e melhor desempenho avaliado.

Não há evidências de que após a experiência universitária o mesmo indivíduo tenha migrado de um nível para outro. No entanto, é possível afirmar que a distribuição atual dos alunos em relação ao seu desempenho é similar entre calouros e veteranos.

Essa afirmação encontra respaldo na tabela ANOVA efeito fixo com P-valor $=0,4409$, a partir da qual não foram encontradas evidências estatísticas para se afirmar que há variação do valor EDTEC com relação ao semestre cursado. Estatisticamente, o desempenho dos alunos é semelhante entre todos os semestres coletados, rejeitando a hipótese de diferenças

(C) ETD-Educação Temática Digital Campinas, SP $\quad$ v.21 n.1 $\quad$ p.182-201 jan./mar. 2019 
após a experiência universitária. Isso sugere que a experiência universitária pouco contribuiu para a ampliação do desempenho em tecnologias para fins educacionais na população pesquisada.

Foi realizado teste ANOVA efeito fixo para a variável turno em relação a EDTEC. Foi identificado $\mathrm{P}$-valor $=0,0159$, suportando a hipótese que há diferença entre os turnos integral e noturno. O turno noturno possui um desempenho ligeiramente superior em relação ao integral.

Uma das possibilidades para essa diferença é a realização de capacitações na área de informática. A distribuição de respostas para a pergunta "Você faz ou já fez curso de Informática?" está descrita na tabela 2:

Tabela 2. Alunos que realizaram curso de informática por turno

\begin{tabular}{lccc}
\hline & Integral & Noturno & Total (\%) \\
\hline Nunca fiz & 79 & 50 & $129(42 \%)$ \\
Pretendo fazer & 13 & 13 & $26(8 \%)$ \\
Estou fazendo & 6 & 2 & $8(3 \%)$ \\
Já fiz & 62 & 83 & $145(47 \%)$ \\
\hline Total & 160 & 148 & $308(100 \%)$ \\
\hline
\end{tabular}

Fonte: dados da pesquisa.

Os dados revelam que, entre os alunos do período integral, $49 \%$ deles nunca fizeram curso na área de informática e $38 \%$, sim. No período noturno, $56 \%$ relatam já terem realizado algum curso de informática e apenas 33\%, nunca.

O teste ANOVA efeito fixo para a variável "curso de informática" em relação a EDTEC suporta a hipótese da ocorrência de diferença motivada por essa situação, com P-valor = 0,0019 .

A comparação entre as médias para buscar identificar se existe diferença estatisticamente relevante entre elas foi feita por meio do teste de Tukey (TSD - Tukey Significant Difference), o resultado identifica três grupos distintos em relação a EDTEC e a realização de cursos na área de TI. Há aqueles que já cursaram e estão em nível mais alto (média 0,67), em seguida estão os alunos que sentem necessidade de capacitação nesse

(C) ETD-Educação Temática Digital Campinas, SP $\quad$ v.21 n.1 $\quad$ p.182-201 jan./mar. 2019 
sentido (média 0,65) e por último, no mesmo grupo, estão aqueles que nunca fizeram (média 0,62 ) ou estão fazendo (média 0,56).

A revelação de que o grupo com pior média apurada está buscando por meios próprios aprimorar-se é positivo do ponto de vista da capacidade de se perceber em desvantagem e por si mesmo buscar mecanismos para melhoria pessoal; essa condição pode indicar uma oportunidade a ser preenchida pela instituição em atividades de extensão, que entre outras finalidades suprem deficiências exigidas no curso superior e colaboram na diminuição da insatisfação e, consequentemente, abandono do curso (GILIOLI, 2016).

A avaliação da hipótese (H3) é importante, pois há estudos que sinalizam que a falta de autoconfiança na utilização dos recursos computacionais gera, em alguns jovens e adultos, ansiedade (CAZAN; COCORADA; MAICAN, 2016).

A pressão por resultados acadêmicos e a dificuldade em alcançá-los é uma das causas de evasão escolar, o desconforto traz impacto maior, principalmente nos semestres iniciais da graduação (BARDAGI; HUTZ, 2014). "A cultura digital tem potencial para transformar as escolas em ambientes de aprendizagem ativos, nos quais os alunos sejam capazes de construir o conhecimento" (PAULA; VALENTE, 2016, p. 10).

Alunos que não se sentem parte dessa cultura tem dificuldades de adaptação. Para Ricoy (2013), limitações econômicas e/ou competências digitais geram um abismo entre os calouros e as instituições poderiam oferecer equipamentos, infraestrutura e cursos extras para reduzir essas assimetrias.

Nas universidades públicas brasileiras há ingresso por cotas para alunos de baixa renda (BRASIL, 2012). O Programa Nacional de Assistência Estudantil (PNAES) estabelece como objetivo a redução da taxa de retenção e evasão e para isso estabelece que devem ser realizadas ações de inclusão digital (BRASIL, 2010). Porém, os cotistas não influenciam o índice de evasão (VIEIRA et al., 2007) e a legislação brasileira determina que a inclusão digital deve ser universal, em todos os níveis de ensino e integrada a outras práticas educacionais (BRASIL, 2014).

A ampliação desse conceito extrapola o fornecimento de um computador e seus aspectos técnicos e direciona-se no sentido da participação social, cidadania digital, desenvolvimento humano e sustentabilidade (PNUD, 2001; ANDRADE-DUVERNOY; RÉGNIER, 2012; UNESCO, 2015).

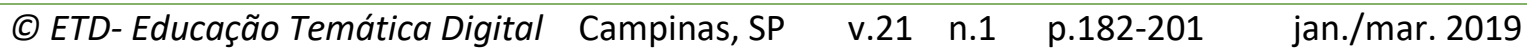


Com o propósito de avaliar a hipótese (H3), observou-se as respostas para a questão "Eu me sinto desconfortável ou inseguro para utilizar o computador para estudar.", dispostas em uma escala Likert em que a respostas "nunca", "às vezes", "muitas vezes" e "sempre" respectivamente equivalem a 1, 2, 3 e 4 .

A figura 3 apresenta os resultados por meio do gráfico de intervalo de confiança das médias em relação ao número de semestres cursados:

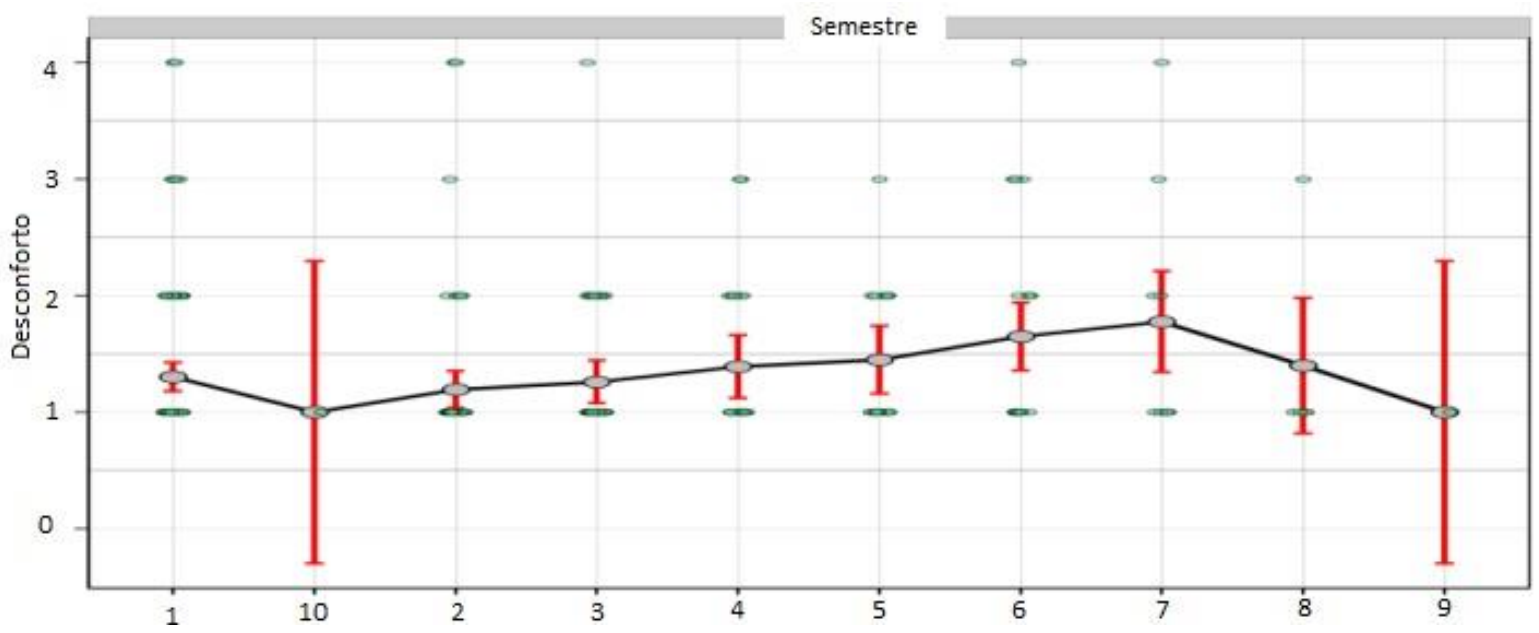

Figura 3 - Intervalo de confiança das médias para desconforto por semestre Fonte: dados da pesquisa

Essa informação revela que no geral, o desconforto ou insegurança são baixos, situados entre "nunca" e "algumas vezes". No oitavo, nono e décimo semestres há no total apenas 7 participantes, consequentemente o intervalo de confiança ficou ampliado e impõe limitações a uma análise mais precisa. Importante destacar a existência de outliers em vários semestres, que relataram sempre sentir desconforto ou insegurança no uso do computador. Conforme apurado pelo teste de Kruskal-Wallis, há diferenças estatisticamente significativas (a um nível de significância de 0,05) entre os semestres e o nível de insegurança ou desconforto na utilização do computador para os estudos. A hipótese (H3) foi rejeitada, pois o desconforto possui tendência de alta em relação aos semestres cursados.

Isso sugere novas pesquisas sobre o assunto, pois o dado pode estar relacionado a dificuldades em acessar conteúdos mais específicos do curso, cansaço pela rotina de estudos, questões de acessibilidade ou uso inadequado das tecnologias digitais. Esse incômodo pode afastar o aluno de uma ferramenta útil para seus estudos e também deve ser considerado nas estratégias educacionais que envolvam recursos computacionais, uma vez que "Educar é 
substantivamente formar. Divinizar ou diabolizar a tecnologia ou a ciência é uma forma altamente negativa e perigosa de pensar errado" (FREIRE, 1996, p. 19).

Com o propósito de avaliar a hipótese (H4), a investigação mais detalhada em como se comporta a utilização da tecnologia para fins educacionais, mostra que há desempenho diferente nos fatores observados. Para identificar o fator que apresenta o maior nível relatado em relação aos demais, foi utilizado o teste proposto por HSU (Multiple Comparisons with the Best-MCB) com intervalo de confiança 95\%, que nos informa que o desempenho em "ferramentas básicas e comunicação" é superior aos demais, e que não há evidência estatística para se rejeitar a hipótese que os fatores solução de problemas e produtividade sejam diferentes, ainda que a média deste seja menor que daquele. Isso confirma a hipótese 4.

Uma das razões para o desempenho superior no fator ferramentas básicas e comunicação está relacionado com redes sociais, aplicativos de comunicação tais como Whatsapp, Messenger, utilização de editores de texto, e-mail e navegação na internet, muito comuns entre os jovens brasileiros (SOUSA; LEÃO, 2016). Por outro lado, os fatores produtividade e solução de problemas demandam letramento digital mais aprofundado, com assuntos relacionados, por exemplo, a planilhas eletrônicas, criação de apresentações, noções de marketing digital, categorização de informação, configuração de hardware, software e segurança da informação. A aprendizagem desses assuntos é menos intuitiva e é comumente encontrada em cursos de informática ou ambientes profissionais, porém pouco explorada no cotidiano de universitários ou incentivada pelos seus professores (SILVA et al., 2014). A qualificação em tecnologias digitais deve atingir também gestores e professores, que são as naturais lideranças em ambiente escolar (CARDOSO, 2015).

A avaliação sobre a percepção dos estudantes sobre incentivo dos professores para utilizar o computador para estudar e disponibilidade de infraestrutura favoráveis (H5) justifica-se, pois, muitas iniciativas de ampliação da informatização falharam porque não consideraram o processo pedagógico, apresentaram falhas na condução da capacitação dos professores ou não existiam condições de infraestrutura aderentes às expectativas didáticas de utilização (CGI.BR, 2016). Os resultados estão apresentados na tabela 3. 
Tabela 3. Percepção de incentivo e condições de infraestrutura

\begin{tabular}{lcc}
\hline & Infraestrutura (\%) & Incentivo (\%) \\
\hline Nunca & $7(2 \%)$ & $32(10 \%)$ \\
Às vezes & $58(19 \%)$ & $139(45 \%)$ \\
Muitas vezes & $134(44 \%)$ & $101(33 \%)$ \\
Sempre & $109(35 \%)$ & $36(12 \%)$
\end{tabular}

Fonte: dados da pesquisa.

A hipótese (H5) foi confirmada e o teste Kruskal-Wallis indica que os grupos incentivo e infraestrutura são estatisticamente diferentes.

A utilização de mídias digitais no ambiente de trabalho é essencial na maioria das profissões da atualidade. A universidade deve estar coerente com essa realidade, o desafio é integrar a tecnologia da educação em seu contexto institucional. "A falta de treinamento formal para os professores está sendo compensada pela aprendizagem informal e pelo desenvolvimento profissional para fins específicos" (FREEMAN; ADAMS BECKER; HALL, 2015, p. 7). Docentes tendem a reproduzir as técnicas por meio das quais aprenderam na sua própria formação, evidenciando "tensões no espaço escolar em relação a como se relacionar com as novas tecnologias" (REIS, 2014, p. 1202). Há décadas atrás, Papert (1973, p.30) visualizava formas pelas quais a tecnologia poderia aprimorar a educação, e classificava as maneiras como as escolas interagiam com esses recursos em reformistas (que as incorporam sem alteração da natureza), intermediárias (fazem a suplementação por meio de centros de aprendizagem específicos) e revolucionárias (que introduzem uma alternativa diferente às conhecidas). Esse desafio perdura há décadas e permanece em processo de superação (PAPERT, 1993; CGI.BR, 2016).

As iniciativas com modelos de aprendizagem inovadores muitas vezes buscam apoiarse em infraestrutura desatualizada ou limitada, uma vez que a gestão dos serviços de tecnologia está desalinhada às necessidades da área acadêmica, focada nos serviços administrativos ao invés do tripé ensino-pesquisa-extensão. Considerava-se, em ambiente escolar, que a utilização do computador nos estudos deveria ser fortalecida nos laboratórios de informática, onde em geral a internet é melhor, porém essa estratégia vem se enfraquecendo com o sucateamento dos equipamentos (CGI.BR, 2016) e pela utilização de dispositivos pessoais portáteis, principalmente notebooks, tablets e smartphones, que

(C) ETD-Educação Temática Digital Campinas, SP $\quad$ v.21 n.1 $\quad$ p.182-201 jan./mar. 2019 
conferem mobilidade para utilização de recursos computacionais em qualquer sala de aula que possua internet wireless disponível (TWYMAN; HEWARD, 2016).

\section{CONSIDERAÇÕES FINAIS}

Foi possível constatar que os universitários percebem os recursos computacionais como otimizadores de seu processo de aprendizagem, porém, possuem desempenho diferente em sua apropriação para aprendizagem.

A imersão digital em que se encontra a sociedade atual favorece atitudes de informação e comunicação, em que os discentes em idade universitária destacam-se de maneira uniforme. Porém, nas aplicações das TDIC para solução de problemas ou melhorias de produtividade há grande variação, independente se é calouro ou veterano. Isso sugere que a capacitação para uso de TDIC na aprendizagem não está suportada em políticas educacionais na educação básica ou superior e homens e mulheres dependem de iniciativas extracurriculares, tais como cursos de informática, para esse empoderamento.

Há um abismo na utilização de tecnologia para fins educacionais e um dos desafios nas universidades brasileiras é a evasão e retenção escolar, em que o desempenho acadêmico insatisfatório influencia nesse sentido. Na medida em que os alunos relatam que aprendem melhor com uso de computadores, processos didáticos que integrem recursos presentes nas tecnologias digitais de informação e comunicação podem ser promissores para superá-lo.

Sugere-se que essa lacuna seja amenizada com atividades extracurriculares de nivelamento tecnológico.

No tripé "professores-alunos-tecnologia", há aqueles que não experimentaram tecnologia didática durante sua formação, quando internet brasileira estava em estágios iniciais de desenvolvimento; universitários que conhecem tecnologia de informação para fins educacionais em nível básico, voltada principalmente para comunicação; e serviços de infraestrutura tecnológicas desarticuladas da atividade fim ensino-pesquisa-extensão.

O discente de nível universitário, ao tomar decisões sobre o que aprender e sobre o que publicar, quais canais utilizar e quais conexões estabelecer, adquire autonomia operacional para manter-se atualizado profissionalmente, colaborar com os colegas, realizar publicações científicas e exercer sua cidadania.

O engajamento e integração social promovido pela educomunicação desenvolvem a cidadania global, um dos objetivos mundiais da agenda 2030 da Unesco. Seu arcabouço

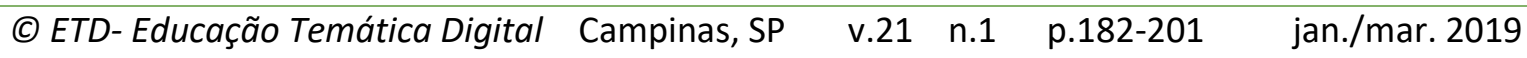


teórico e experiências práticas podem ser aproveitadas para capacitação de professores e para atividades de ensino, pesquisa e extensão com os alunos, pois articula o processo educativo em conjunto com a intencionalidade da comunicação. Consequente ao aumento da utilização de recursos de TDIC na área didática, a complexidade de seu gerenciamento nessas instituições ampliar-se-á, exigindo um modelo interdisciplinar semelhante ao papel do educomunicador (SÃO PAULO, 2015).

Há instituições com iniciativas na estruturação de órgãos específicos com a finalidade de apoiar e garantir a qualidade do uso educacional de tecnologias (LEÃO; RODRIGUES, 2015). Tais experiências, entre eles o Núcleo de Informática Aplicada à Educação na Unicamp (criado em 1983), o Laboratório de Informática na Educação da Universidade de São Paulo (criado em 1996) e o Núcleo de Tecnologias Educacionais da Universidade Federal do ABC (criado em 2016), podem ser acompanhadas para eventual disseminação entre outras instituições de ensino superior (UNICAMP, 1983; UFABC, 2016; USP, 2017).

Este estudo encontrou outros questionamentos, entre eles "Por que a insegurança e desconforto no uso de TDIC apresentam tendência de alta à medida que se avançam os semestres?". Novas pesquisas são necessárias, inclusive sobre eventual relação entre a autonomia operacional, relacionada ao conhecimento técnico, com autonomia moral, que aponta no sentido de como essa imersão em idades cada vez mais precoces na cibercultura, pode influenciar o processo de desenvolvimento moral do indivíduo.

Em adição a pesquisas acadêmicas sobre o assunto, possivelmente a criação de espaços de troca para professores, alunos e administradores poderia lançar pilares para o aperfeiçoamento do cuidado com o próximo e para cidadania digital. As instituições de ensino têm se adaptado, isso é natural uma vez que a "escola existe para formar sujeitos preparados para sobreviver nesta sociedade" (LIBÂNEO; SANTOS, 2010, p. 17).

\section{REFERÊNCIAS}

ANDRADE-DUVERNOY, Doriele; RÉGNIER, Jean-Claude. A educomunicação como princípio indissociável da extensão universitária, do protagonismo juvenil e da coesão social: o caso da rede coque vive. Poiésis, v. 5, n. especial, p. 149-164, 2012.

ARRUDA, Juliana S.; FILHO, José A. C.; SIQUEIRA, Liliane R.C. O uso de tecnologias digitais na facilitação da emergência de zonas de desenvolvimento proximal em sala de aula. In: $V$ Congresso Brasileiro de Informática na Educação. 2016. Anais..., Uberlândia: CBIE, 2016. P. 771-780.

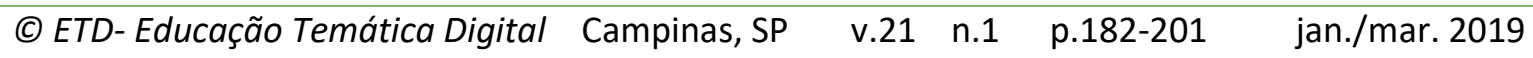


BARDAGI, Marucia; HUTZ, Claudio S. Evasão universitária e serviços de apoio ao estudante: uma breve revisão da literatura brasileira. Psicologia Revista. São Paulo, v. 14, n. 2, p. 279301, 2014.

BÉVORT, Evelyne; BELLONI, M. Luiza. Mídia-educação: conceitos, história e perspectivas. Educação e sociedade. Campinas, v. 30, n. 109, p. 1081-1102, 2009.

BRASIL. Decreto no 7234, de 19 de julho de 2010. Dispõe sobre o Programa Nacional de Assistência Estudantil (PNAES). Diário Oficial da República Federativa do Brasil, Brasília, DF, 20 jul. 2010. Seção 1, p. 5, 2010.

BRASIL. Lei no 12711, de 29 de agosto de 2012. Dispõe sobre o ingresso nas universidades federais e nas instituições de ensino técnico de nivel médio. Diário Oficial da República Federativa do Brasil, Brasília, DF, 30 ago. 2012. Seção 1, p. 1-3, 2012.

BRASIL. Lei no 12965, de 23 de abril de 2014. Estabelece princípios, garantias, direitos e deveres para o uso da Internet no Brasil. Diário Oficial da República Federativa do Brasil, Brasília, DF, 24 abr. 2014. Seção 1, p. 1, 2014.

CAZAN, Ana M.; COCORADA, Elena; MAICAN, Catalin I. Computer anxiety and attitudes towards the computer and the internet with Romanian high-school and university students. Computers in Human Behavior, v. 55, p. 258-267, 2016.

CARDOSO, A. O. C. Tecnologias digitais, currículo e interdisciplinaridade na escola: um link possível a partir da ação docente. Educação por escrito. Porto Alegre, v. 6, n. 2, p. 208-219, 2015.

CAVASSANI, Tiago B.; ANDRADE, Joana J. Você tem face?: perspectivas discentes e implicações do (não) uso do facebook no ensino superior. Educação Temática Digital. Campinas, v.18, n.1, p. 227-247, 2016.

CGI.BR. Educação e tecnologias no Brasil: um estudo de caso longitudinal sobre o uso das tecnologias de informação e comunicação em 12 escolas públicas. São Paulo: Comitê Gestor da Internet no Brasil, 2016.

COCHRAN, Willian G. Sampling techniques. Wiley India Limited, 2007.

CONTE, Elaine; MARTINI, Rosa M. F. As Tecnologias na Educação: uma questão somente técnica? Educação \& Realidade. Porto Alegre, v. 40, n. 4, p. 1191-1207, 2015.

EQUIPE ESTATCAMP. Software Action, versão 3.2. São Carlos, SP: Estatcamp - Consultoria em estatística e qualidade, 2014.

(C) ETD-Educação Temática Digital Campinas, SP $\quad$ v.21 n.1 $\quad$ p.182-201 jan./mar. 2019 
FREEMAN, Alex; ADAMS BECKER, Samantha; HALL, C. 2015 NMC Technology Outlook for Brazilian Universities: A Horizon Project Regional Report. Austin, Texas: The New Media Consortium, 2015.

FREIRE, Paulo. Pedagogia da autonomia: saberes necessários à prática educativa. São Paulo: Paz e terra, 1996.

GADOTTI, Moacir. Perspectivas atuais da educação. São Paulo em Perspectiva. São Paulo, v. 14, n. 2, p. 03-11, 2000.

GILIOLI, Renato S. P. Evasão em Instituições Federais de ensino superior no Brasil: expansão da rede, Sisu e desafios. Estudo técnico. Consultoria Legislativa da Câmara dos Deputados, Brasília-DF: 2016. Disponível em: https://goo.gl/JFzUw8 . Acesso em: 15 nov. 2017.

GROS, Begona; GARCIA, Iolanda; ESCOFET, Anna. Beyond the Net Generation Debate: a comparison of digital learners in face-to-face and virtual universities. International Review of Research in Open and Distance Learning, v. 13, n. 4, p. 190-210, 2012.

INEP. Censo da educação superior 2013: resumo técnico. Brasília, 2015. Disponível em: https://goo.gl/awc9cA . Acesso em: 15 nov. 2017.

ISTE. National Educational Technology Standards (NETS): learning to use technology. Arlington, Estados Unidos, 1998. Disponível em: https://goo.gl/5BVktk. Acesso em: 15 nov. 2017.

JONES, Chris; RAMANAU, Ruslan; CROSS, Simon; HEALING, Graham. Net generation or digital natives: is there a distinct new generation entering university? Computers and Education, v. 54, n. 3, p. 722-732, 2010.

LAPPONI, Juan C. Estatística usando Excel. 4. Ed., Rio de Janeiro: Elsevier, 2005.

LEÃO, M. Izabel de A.; RODRIGUES, José M. A internet como ferramenta de apoio a educomunicação. Núcleo de comunicação e educação da USP.São Paulo, v. 1, p.1-17, 2015.

LEVY, Pierre. Cibercultura. Tradução de Carlos Irineu da Costa. São Paulo: Ed. 34, 1999.

LIBÂNEO, José C.; SANTOS, Akiko. Educação na era do conhecimento em rede e transdisciplinaridade. 2.ed. Campinas: Alinea, 2010.

MARCELA, Paola; DEL, Hermosa. Influencia de las tecnologías de información y comunicación (TIC) en el proceso enseñanza-aprendizaje: una mejora de las competencias digitales. Revista Científica General José María Córdova. Bogotá, v. 13, n.16, p. 121-132, 2015.

(C) ETD- Educação Temática Digital Campinas, SP $\quad$ v.21 n.1 $\quad$ p.182-201 jan./mar. 2019 
PALFREY, John; GASSER, Urs. Born Digital: understanding the first generation of digital natives. New York: Basic Books, 2008.

PAPERT, Seymour. Uses of technology to enhance education. Massachusetts Institute of Technology. Artificial Intelligence Laboratory, Cambridge, 1973. Disponível em: https://dspace.mit.edu/handle/1721.1/6213 . Acesso em 15 nov. 2017.

PAPERT, Seymour. The children's machine: rethinking school in the age of the computer. New York: Basic books, 1993.

PAULA, Bruno H.; VALENTE, José A. Jogos digitais e educação: uma possibilidade de mudança da abordagem pedagógica no ensino formal. Revista Iberoamericana de Educación, v. 70, n. 1, p. 9-28, 2016.

PNUD. Relatório do Desenvolvimento Humano 2001. Fazer as novas tecnologias trabalhar para o desenvolvimento humano. Programa das Nações Unidas para o Desenvolvimento, 2001.

PRENSKY, Marc. Digital natives, digital immigrants. On the Horizon, v. 9, n. 5, p. 1-6, 2001.

REGO, Tereza C. VYGOTSKY: Uma perspectiva histórico-cultural da educação. 21. Ed. Petrópolis, RJ: Editora Vozes, 2010.

REIS, Rosemeire. Aprender na atualidade e tecnologias: implicações para os estudos no ensino médio. Educação \& Realidade. Porto Alegre, v. 39, n. 4, p. 1185-1207, 2014.

RICOY, Carmen; FELIZ, Tiberio; COUTO, Maria J. The digital divide among University Freshmen. Turkish Online Journal of Educational Technology - TOJET. Sakarya, v. 12, n. 2, p. 262-268, 2013.

ROBERTO, Magda S.; FIDALGO, Antônio; BUCKINGHAM, David. De que falamos quando falamos de infoexclusão e literacia digital? Perspetivas dos nativos digitais. Observatorio. Palmas, v. 9, n. 1, p. 043-054, 2015.

SAMPIERI, Roberto H.; COLLADO, Carlos. F.; LUCIO, Maria del P. B. Metodologia de pesquisa. 5. Ed., Porto Alegre-RS: Penso, 2013.

SÃO PAULO. Deliberação do conselho estadual de educação. Aprova o pedido de reconhecimento do Curso de Licenciatura em Educomunicação, da USP. Diário Oficial do Estado. São Paulo, 9 abr. 2015, p. 33, 2015.

SAVIANI, Dermeval. História das idéias pedagógicas no Brasil. 2. Ed. Campinas-SP: Autores associados, 2008.

(C) ETD- Educação Temática Digital Campinas, SP $\quad$ v.21 n.1 $\quad$ p.182-201 jan./mar. 2019 
SILVA, Bento D.; ARAÚJO, Alexandra M.; VENDRAMINI, Claudete; MARTINS, Ronei X; PIOVEZAN, Nayane M.; PRATES, Eli; DIAS, Anelise S; ALMEIDA, Leandro; JOLY, M. Cristina R. Aplicação e uso de tecnologias digitais pelos professores do ensino superior no Brasil e em Portugal. Educação, Formação \& Tecnologias. Lisboa, v. 7, n. 1, p. 3-18, 2014.

SOARES, Ismar O. Gestão comunicativa e educação: caminhos da educomunicação. Comunicação \& Educação. São Paulo, n. 23, p. 16-25, abr. 2002.

SOUSA, Cirlene C.; LEÃO, Geraldo M. P. Ser jovem e ser aluno: entre a escola e o facebook. Educação \& Realidade. Porto Alegre, v. 41, n. 1, p. 279-302, 2016.

TWYMAN, Janet S.; HEWARD, William L. How to improve student learning in every classroom now. International Journal of Educational Research, n. 2015, p. 1-13, 2016.

UFABC. Resolução Consuni $n^{\circ} 168$ - Dispõe sobre o núcleo de tecnologias educacionais. Santo André-SP, 2016. Disponível em: http://nte.ufabc.edu.br/ . Acesso em 10 out. 2017.

UNICAMP. Portaria GR 139, de 17 de maio de 1983. Cria o Núcleo de Informática Aplicada à Educação (NIED). In: UNICAMP, Apresentação do NIED. Disponível em: http://www.nied.unicamp.br/?q=apresentacao . Acesso em 10 out. 2017.

USP. Laboratório de Informática na Educação (LinE). Disponível em: http://line.ime.usp.br/ Acesso em 10 out. 2017.

UNESCO. Transformando nosso mundo: a agenda 2030 para o desenvolvimento sustentável. Rio de Janeiro, 2015. Disponível em: https://goo.gl/qtDGNP. Acesso em 15 nov. 2017.

UNESCO. Community-based lifelong learning and adult education: adult skills and competencies for lifelong learning. Paris, France: 2016.

VENKATESH, Viswanath; MORRIS, Michael G; DAVIS, Gordon B.; DAVIS, Fred D. User acceptance of information technology: toward a unified view. MIS Quarterly, v. 27, n. 3, p. 425-478, 2003.

VIEIRA, Augusto; SOUZA, Marina M. F.; SILVA, Tatiane P. D. M.; CASTRO, Guilherme C. Análise estatística do perfil dos cotistas que evadiram da UERJ. Cadernos do IME - série estatística da UERJ. Rio de Janeiro, v. 22, p. 31-45, 2007.

Revisão gramatica do texto sob responsabilidade de: Sandra Salviato Terra E-mail: rsterra@uol.com.br 